\title{
Diametral dimension of some pseudoconvex multiscale spaces
}

\author{
by \\ Jean-Marie Aubry (Créteil) and Françoise Bastin (Liège)
}

\begin{abstract}
Stemming from the study of signals via wavelet coefficients, the spaces $S^{\nu}$ are complete metrizable and separable topological vector spaces, parametrized by a function $\nu$, whose elements are sequences indexed by a binary tree. Several papers were devoted to their basic topology; recently it was also shown that depending on $\nu, S^{\nu}$ may be locally convex, locally $p$-convex for some $p>0$, or not at all, but under a minor condition these spaces are always pseudoconvex. We deal with some more sophisticated properties: their diametral dimensions show that they are Schwartz but not nuclear spaces. Moreover, Ligaud's example of a Schwartz pseudoconvex non- $p$-convex space is actually a particular case of $S^{\nu}$.
\end{abstract}

1. Introduction. The theory of signal processing poses numerous challenges to functional analysis. Natural spaces for signals or images often have peculiar properties which in turn play a role in the performance of signal processing algorithms.

Since the introduction of wavelets in the 80's, the representation of a signal by means of its wavelet coefficients has been a widely used tool. From a functional analysis viewpoint, a property of signals which has an expression in terms of wavelet coefficients independent of the chosen wavelet basis can be intrinsically studied using sequence spaces. For instance, the pointwise Hölder exponents of a function can be characterized by means of its wavelet coefficients under a mild global regularity hypothesis. Pointwise regularity properties, for instance multifractality, ultimately depend only on the distribution of the wavelet coefficients.

A key feature of wavelets is that the coefficients are inherently indexed in a hierarchical (multiscale) way, most often on a binary tree. Adapted sequence spaces should mirror this organization. For instance the Besov norms (Definition 2) have an equivalent expression in terms of wavelet coefficients that clearly uses the multiscale structure. Nevertheless, the Besov framework

2010 Mathematics Subject Classification: 46A16, 46A45, 46A11, 46A13.

Key words and phrases: pseudoconvex space, nuclearity, diametral dimension. 
was not sufficiently precise to handle all the accurate information contained in the distribution of the wavelet coefficients. In this context, spaces of type $S^{\nu}$ were introduced by Jaffard [8] and studied in previous papers by Dispa, Jaffard and the present authors [1, 3]. These sequence spaces are new, stem from practical problems in multifractal analysis and have non-trivial topological properties. In this paper we wish to answer previously open questions, including those of J. Wengenroth and A. Pełczyński about nuclearity and diametral dimensions of these spaces.

1.1. $S^{\nu}$ spaces. Let us briefly summarize the definitions and main properties established in our previous articles. We consider sequences of complex numbers indexed by the set $\Lambda:=\bigcup_{j \in \mathbb{N}_{0}}\{j\} \times\left\{0, \ldots, 2^{j}-1\right\}$ where $\mathbb{N}_{0}:=\{0\} \cup \mathbb{N}$. These can for instance be sequences of wavelet coefficients of periodic distributions defined on $\mathbb{T}:=\mathbb{R} / \mathbb{Z}$, in an appropriate wavelet basis.

Definition 1. The asymptotic profile of a sequence $x \in \mathbb{C}^{\Lambda}$ is the function $\nu_{x}$ defined on $\mathbb{R}$ by

$$
\nu_{x}(\alpha):=\lim _{\varepsilon \rightarrow 0^{+}} \limsup _{j \rightarrow \infty} \frac{\log \left(\#\left\{k:\left|x_{j, k}\right| \geq 2^{-(\alpha+\varepsilon) j}\right\}\right)}{\log \left(2^{j}\right)}
$$

The role of the asymptotic profile of wavelet coefficients in multifractal analysis emerged in [2, 4] following the so-called large deviations multifractal formalisms [6, 13, 17].

It is easily seen that $\nu_{x}$ is always a non-decreasing right-continuous function with values in $\{-\infty\} \cup[0,1]$. Conversely, given an arbitrary function $\nu$ enjoying these properties, not identically equal to $-\infty$ (we then say that $\nu$ is an admissible profile), we define

$$
S^{\nu}:=\left\{x: \nu_{x}(\alpha) \leq \nu(\alpha) \forall \alpha \in \mathbb{R}\right\} .
$$

Roughly speaking, a sequence $x$ belongs to $S^{\nu}$ if (asymptotically) at each scale $j$ the number of $k$ such that $\left|x_{j, k}\right| \geq 2^{-\alpha j}$ is less than $2^{\nu(\alpha) j}$. It is a complex linear space which can be endowed with a unique metrizable topology that makes it a complete, separable topological vector space and that is stronger than the product topology (of coordinatewise convergence for sequences). We shall explain in $\$ 2.1$ and $\$ 4$ how this topology can be described.

In this context, many questions of functional analysis appear, the most elementary of which is local convexity. We established in [1] that $S^{\nu}$ is locally $p_{0}$-convex (and not better) if $p_{0}>0$, where $p_{0}$ is the "minimal slope" of $\nu$, more precisely

$$
p_{0}:=\min \left(1, \inf _{0 \leq \nu(\alpha)<1} \underline{\partial}^{+} \nu(\alpha)\right),
$$


where we have used the right-inf derivative notation

$$
\underline{\partial}^{+} \nu(\alpha):=\liminf _{h \rightarrow 0^{+}} \frac{\nu(\alpha+h)-\nu(\alpha)}{h} .
$$

The case $p_{0}=1$ corresponds to a locally convex space, therefore a Fréchet space. However, with applications to multifractal analysis in mind, there is no reason to focus on this particular case, because $\nu$ is closely related (see [2, 7, 8]) to a spectrum of singularities for which the minimal slope $p_{0}$ could very well be strictly smaller than 1 ; on the contrary, we have a new motivation to be interested in $p$-convex spaces.

1.2. Outline of the results. We start in $\$ 2$ with the description of the $S^{\nu}$ topology and its relationship to Besov $(p-)$ norms. In $\$ 3$ we recall the definitions of nuclearity and diametral dimensions, how they are related and what happens when the spaces are only locally $p$-convex instead of locally convex. The main result is the computation of the diametral dimension of $S^{\nu}$ in Theorem 1. As a corollary, we shall see that $S^{\nu}$ is in fact a Schwartz space, but not nuclear (it was already proved in [3] that all bounded sets in $S^{\nu}$ are relatively compact, so it is a Montel space, Fréchet-Montel in the case $p_{0}=1$ ). The last $\$ 4$ deals with the case $p_{0}=0$, i.e. local pseudoconvexity; on this occasion we revisit Ligaud's example of a Schwartz pseudoconvex space.

\section{2. $S^{\nu}$ topology}

2.1. Sum of Besov spaces. Let us assume for the moment that $p_{0}>0$. In that case, the topology of $S^{\nu}$ can be described by a set of $p_{0}$-norms fitting the sum of two Besov spaces. Originally the topology was defined in [3] using distances that cover the case $p_{0}=0$ as well, but $p_{0}$-norms are easier to manipulate and hence we prefer to start from them. A description using $p$-norms, adapted to the case $p_{0}=0$, of this topology will be presented in $\$ 4$

Let us first recall the definition of Besov quasinorms.

Definition 2. For $s \in \mathbb{R}, 0<p<\infty$, the $b_{p, \infty}^{s}$ Besov quasinorm (norm if $p \geq 1$ ) of a sequence $x$ is given by

$$
\|x\|_{b_{p, \infty}^{s}}:=\sup _{j \in \mathbb{N}_{0}} 2^{s j}\left(2^{-j} \sum_{k=0}^{2^{j}-1}\left|x_{j, k}\right|^{p}\right)^{1 / p}
$$

and if $p=\infty$,

$$
\|x\|_{b_{\infty, \infty}^{s}}:=\sup _{j \in \mathbb{N}_{0}} \sup _{0 \leq k<2^{j}} 2^{s j}\left|x_{j, k}\right| .
$$

For $\alpha, s \in \mathbb{R}$, we define the $p_{0}$-norm on the sum space $b_{p_{0}, \infty}^{s}+b_{\infty, \infty}^{\alpha}$ by

$$
\|y\|_{\alpha, s}:=\inf \left\{\left\|y^{\prime}\right\|_{b_{\infty, \infty}^{\alpha}}+\left\|y^{\prime \prime}\right\|_{b_{p_{0}, \infty}^{s}}: y^{\prime}+y^{\prime \prime}=y\right\} .
$$


For coherence of notation we also include the case $s=\infty$ and write

$$
\|y\|_{\alpha, \infty}:=\|y\|_{b_{\infty, \infty}^{\alpha}} .
$$

Let $\nu$ be an admissible profile and $S^{\nu}$ be defined as in the introduction by (2). Two important parameters are

$$
\alpha_{\min }:=\inf \{\alpha: \nu(\alpha) \geq 0\} \quad \text { and } \quad \alpha_{\max }:=\inf \{\alpha: \nu(\alpha)=1\},
$$

both necessarily finite if $p_{0}>0$. Now let

$$
\mathbb{U}:=\left\{(A, \varepsilon): A:=\left\{\alpha_{1} \leq \cdots \leq \alpha_{L}\right\} \subset\left(-\infty, \alpha_{\max }\right), \varepsilon>0\right\} .
$$

Any $(A, \varepsilon) \in \mathbb{U}$ determines the $p_{0}$-norm

$$
\|x\|_{A, \varepsilon}:=\sup _{1 \leq l \leq L}\|x\|_{\alpha_{l}-\varepsilon, \alpha_{l}-\varepsilon+\left(1-\nu\left(\alpha_{l}\right)\right) / p_{0}} .
$$

Then we set

$$
\mathcal{U}:=\left\{B_{A, \varepsilon}(r):(A, \varepsilon) \in \mathbb{U}, r>0\right\}
$$

where $B_{A, \varepsilon}(r):=\left\{x:\|x\|_{A, \varepsilon} \leq r\right\}$. The fact that $\mathcal{U}$ is a 0 -basis of $S^{\nu}$ follows directly from [1, Theorem 2] and the following comparisons: on one hand we clearly have

$$
\|y\|_{\alpha-\varepsilon, s} \leq\|y\|_{\alpha, s}
$$

and on the other hand,

$$
\|y\|_{\alpha, \alpha-\varepsilon+(1-\nu(\alpha)) / p_{0}} \leq\|y\|_{\alpha^{\prime}-\varepsilon^{\prime}, \alpha^{\prime}-\varepsilon^{\prime}+\left(1-\nu\left(\alpha^{\prime}\right)\right) / p_{0}}
$$

if $\alpha^{\prime}>\alpha$ is such that $\nu\left(\alpha^{\prime}\right) \leq \nu(\alpha)+\varepsilon p_{0}$ and $\varepsilon^{\prime}=\alpha^{\prime}-\alpha$.

The special multiscale structure of these norms also implies the following.

Lemma 2.1. For any $A$ and $\varepsilon>\varepsilon^{\prime}>0$,

(i) if $j<j_{0}$ implies $x_{j, k}=0$, then $\|x\|_{A, \varepsilon} \leq 2^{\left(\varepsilon^{\prime}-\varepsilon\right) j_{0}}\|x\|_{A, \varepsilon^{\prime}}$;

(ii) if $j>j_{0}$ implies $x_{j, k}=0$, then $\|x\|_{A, \varepsilon} \geq 2^{\left(\varepsilon^{\prime}-\varepsilon\right) j_{0}}\|x\|_{A, \varepsilon^{\prime}}$.

Proof. The inequalities are easily checked on the $b_{\infty, \infty}^{\alpha-\varepsilon}$ and $b_{p, \infty}^{s-\varepsilon}$ norms, hence on the norms $\|\cdot\|_{\alpha-\varepsilon, s-\varepsilon}$, and the lemma is proved.

2.2. Approaching $\nu$. A convenient way to visualize the norms involved (but not to write proofs!) is to associate to a sequence space $X \subset \mathbb{C}^{\Lambda}$ its parameter set $\Pi(X)$, which is the set of points $(\alpha, \beta), \alpha \in \mathbb{R}$ and $\beta \in[0,1]$, such that the "typical sequence" having at each scale $j$ a number $\left\lfloor 2^{\beta j}\right\rfloor$ of coefficients equal to $2^{-\alpha j}$ (and the rest equal to 0 ) belongs to $X$. For instance $\Pi\left(S^{\nu}\right)$ is just the hypograph of $\nu$ (that is, the set $\{(\alpha, \beta): 0 \leq \beta \leq \nu(\alpha)\}$ ), whereas

$$
\Pi\left(b_{p, \infty}^{s}\right)=\{(\alpha, \beta): 0 \leq \beta \leq \min (1+(\alpha-s) p, 1)\}
$$

for any $0<p<\infty$, and

$$
\Pi\left(b_{\infty, \infty}^{s}\right)=\{(\alpha, \beta): \alpha \geq s\} .
$$


Clearly $\Pi\left(b_{p_{0}, \infty}^{s}+b_{\infty, \infty}^{\alpha}\right)=\Pi\left(b_{p_{0}, \infty}^{s}\right) \cup \Pi\left(b_{\infty, \infty}^{\alpha}\right)$ and $\Pi\left(X \cap X^{\prime}\right)=$ $\Pi(X) \cap \Pi\left(X^{\prime}\right)$, so for any $(A, \varepsilon) \in \mathbb{U}$, the parameter set of the space normed by $\|\cdot\|_{A, \varepsilon}$, which is an intersection of sum spaces, is the hypograph of the function $\varphi_{A, \varepsilon}$ defined by

$$
\varphi_{A, \varepsilon}(\alpha):=\min \left(1, p_{0}\left(\alpha-\alpha_{l}+\varepsilon\right)+\nu\left(\alpha_{l}\right)\right)
$$

when $\alpha_{l-1} \leq \alpha+\varepsilon<\alpha_{l}$, understanding that $\alpha_{0}:=-\infty$ (see Figure 1). The fact that the intersection of all these (when $(A, \varepsilon)$ ranges over $\mathbb{U}$ ) equals the hypograph of $\nu$ mirrors the fact that $\mathcal{U}$ is a 0 -basis of $S^{\nu}$ (note that $\varphi_{A, \varepsilon} \geq \nu$ when $\varepsilon>0)$.

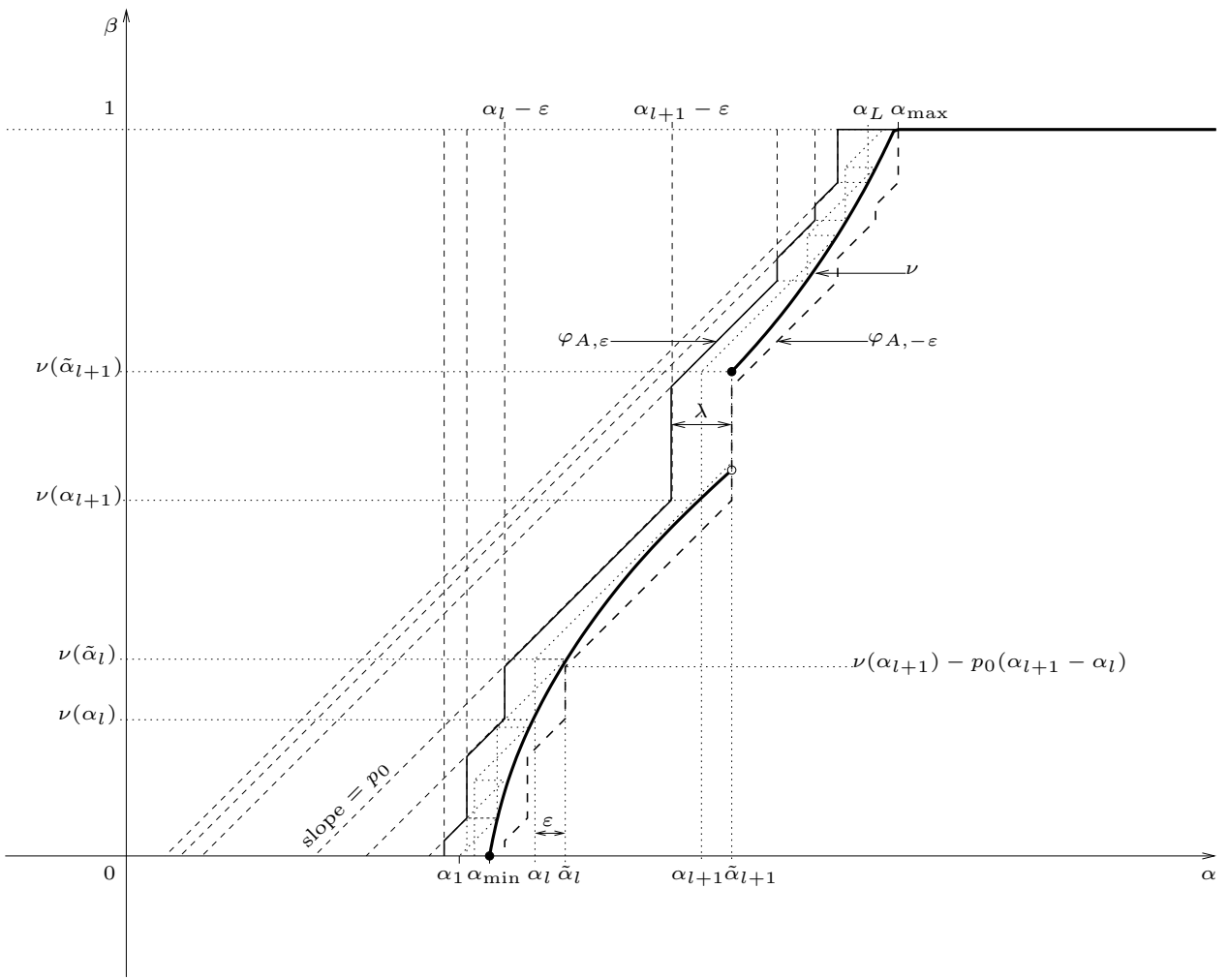

Fig. 1. The construction of $\varphi_{A, \varepsilon}$ in Lemma 2.2 (step $l=5$ )

The following lemma shows that the functions $\varphi_{A, \varepsilon}$ can approach $\nu$ arbitrarily closely from the left (this will be needed in $\$ 3.3$ ).

Lemma 2.2. For any $\lambda>0$, there exists $(A, \varepsilon) \in \mathbb{U}$ such that for all $\alpha \in \mathbb{R}$,

$$
\varphi_{A, \varepsilon}(\alpha) \leq \nu(\alpha+\lambda)
$$


Proof. With $\varepsilon:=\lambda / 2$ we make the following iterative construction: starting with $\tilde{\alpha}_{1}:=\alpha_{\min }$, let

$$
\tilde{\alpha}_{l+1}:=\sup \left\{\alpha: \nu(\alpha)-\nu\left(\tilde{\alpha}_{l}\right)<p_{0}\left(\alpha-\left(\tilde{\alpha}_{l}-\varepsilon\right)\right)\right\}
$$

and we stop, after at most $\left(p_{0} \varepsilon\right)^{-1}$ steps, when reaching an $\tilde{\alpha}_{L} \geq \alpha_{\max }$ (actually $\tilde{\alpha}_{L}=\alpha_{\max }$ or $+\infty$ ), which we replace by $\tilde{\alpha}_{L}:=\alpha_{\max }$. Finally, for $1 \leq l \leq L$ let $\alpha_{l}:=\tilde{\alpha}_{l}-\varepsilon$ and $A:=\left\{\alpha_{1}, \ldots, \alpha_{L}\right\}$.

Let us show that $\varphi_{A, \varepsilon}(\alpha) \leq \nu(\alpha+\lambda)$ for all $\alpha$. Since $\lambda=2 \varepsilon$, this amounts to showing that $\varphi_{A,-\varepsilon}(\alpha):=\varphi_{A, \varepsilon}(\alpha-2 \varepsilon) \leq \nu(\alpha)$. The left-hand side function is increasing, right-continuous, piecewise linear with slope $p_{0}$ and its graph is the union of segments successively joined by the verticals $\left[\left(\tilde{\alpha}_{l}, \nu\left(\alpha_{l}\right)\right),\left(\tilde{\alpha}_{l}, \nu\left(\alpha_{l+1}\right)-p_{0}\left(\alpha_{l+1}-\alpha_{l}\right)\right)\right]$ for $1 \leq l \leq L-1$, then the vertical $\left[\left(\tilde{\alpha}_{L}, \nu\left(\alpha_{L}\right)\right),\left(\tilde{\alpha}_{L}, 1\right)\right]$, and finishing with the half-line $\left[\left(\tilde{\alpha}_{L}, 1\right),(\infty, 1)\right)$ (the graph of $\varphi_{A,-\varepsilon}$ is the dashed line in Figure 1). Note that for $1 \leq l \leq L-1$,

$$
\nu\left(\alpha_{l+1}\right)=\nu\left(\tilde{\alpha}_{l+1}-\varepsilon\right) \leq \lim _{\alpha \rightarrow \tilde{\alpha}_{l+1}^{-}} \nu(\alpha)-p_{0} \lambda=\nu\left(\tilde{\alpha}_{l}\right)+p_{0}\left(\tilde{\alpha}_{l+1}-\tilde{\alpha}_{l}\right)
$$

by construction of $\tilde{\alpha}_{l+1}$ from $\tilde{\alpha}_{l}$. Hence $\nu\left(\alpha_{l+1}\right)-p_{0}\left(\alpha_{l+1}-\alpha_{l}\right) \leq \nu\left(\tilde{\alpha}_{l}\right)$, which suffices to prove the inequality for all $\alpha$.

\section{Nuclearity and diametral dimensions}

3.1. On nuclearity for $p$-convex spaces. Let us temporarily come back to more general topological considerations. Nuclearity for (Hausdorff) locally convex spaces is classically defined in terms of nuclear operators between normed spaces. Recall, from [9] or [16, that an operator $S: E \rightarrow F$ is nuclear if there exist sequences $u_{m}$ and $y_{m}$ bounded respectively in $E^{\prime}$ and $F$, and a summable sequence of complex numbers $\lambda_{m}$ such that for any $x \in E$,

$$
S(x)=\sum_{m} \lambda_{m} u_{m}(x) y_{m} .
$$

A locally convex space $E$ is called nuclear if, for any absolutely convex 0 -neighborhood $U$, there exists an absolutely convex 0-neighborhood $V$ absorbed by $U$ such that the canonical map $E_{(V)} \rightarrow E_{(U)}$ is nuclear (using the standard notation $E_{(U)}:=E /\{x: \forall \rho>0, x \in \rho U\}$ normed by $\left.\|x\|_{U}:=\inf \{\rho>0: x \in \rho U\}\right)$.

If we are to establish (positive or negative) nuclearity properties for the family of spaces $S^{\nu}$, we should first try to extend this notion to locally $p$-convex spaces. A somewhat naïve approach consists in keeping the same definition for $E_{(U)}$, using absolutely $p$-convex neighborhoods $U$ and replacing norms by $p$-norms. But then a nuclear operator in the previous sense would not necessarily be continuous, so this notion has to be changed as well. 
Definition 3. An operator between $p$-normed spaces $E$ and $F$ is said to be $p$-nuclear $\left({ }^{1}\right)$ if there exist sequences $u_{m}$ and $y_{m}$ bounded respectively in $E^{\prime}$ and $F$, and a sequence of complex numbers $\lambda_{m}$ satisfying $\sum_{m}\left|\lambda_{m}\right|^{p}<\infty$ such that (8) holds for any $x \in E$.

A locally $p$-convex space $X$ is called $p$-nuclear if, for any absolutely $p$ convex 0-neighborhood $U$, there exists a 0 -neighborhood $V$ absorbed by $U$ such that the canonical map $E_{(V)} \rightarrow E_{(U)}$ is $p$-nuclear.

Remember that locally convex implies locally $p$-convex, whereas $p$-nuclear is stronger than nuclear. In fact, we have an equivalence which closes this aspect of the question.

Proposition 3.1. For any $0<p \leq 1$, the following are equivalent:

(i) $E$ is locally p-convex and p-nuclear;

(ii) $E$ is locally convex and nuclear.

Proof. (i) $\Rightarrow$ (ii): For any 0-neighborhood $U$ there exists $V$ as above, and for any $x \in V$ we have

$$
\|x\|_{U}^{p}=\left\|\sum_{m} \lambda_{m} u_{m}(x) y_{m}\right\|_{U}^{p} \leq C_{1} \sum_{m}\left|\lambda_{m}\right|^{p}\left|u_{m}(x)\right|^{p} \leq C_{2} \sup _{m}\left|u_{m}(x)\right|^{p},
$$

so $U$ contains a convex 0 -neighborhood determined by the set of seminorms $\left|u_{m}(x)\right|$. This proves that the space is locally convex.

(ii) $\Rightarrow(\mathrm{i})$ : We have to show that we can replace the nuclear operators in the classical definition of nuclearity by the seemingly stronger requirement that the canonical maps are $p$-nuclear. For this we will say, using the terminology of Pietsch [16], that a map $\phi \in \mathcal{L}(E, F)$ is of type $\mathbf{l}^{p}$ if the sequence of its approximation numbers $\alpha_{r}(\phi)$ satisfies $\sum_{r} \alpha_{r}(\phi)^{p}<\infty$. If $E$ is nuclear then for any 0-neighborhood $U=: U_{0}$ and $n \geq 2 / p$ there are $U_{0} \supset \cdots \supset U_{2 n}=: V$ such that each map $\phi_{i}: E_{\left(U_{i}\right)} \rightarrow E_{\left(U_{i-1}\right)}$ is nuclear. Then by [16, 8.4.5] for all $1 \leq i \leq n, \phi_{2 i} \circ \phi_{2 i-1}$ is of type $\mathbf{l}^{2}$, hence, applying [16, 8.2.7], $\phi:=\phi_{2 n} \circ \cdots \circ \phi_{1}: E_{(V)} \rightarrow E_{(U)}$ is of type $\mathbf{l}^{p}$. By [16, 8.4.2], $\phi$ is then $p$-nuclear.

In contrast, the definition of a $S$ chwartz space (where the maps $E_{(V)} \rightarrow$ $E_{(U)}$ are required to be precompact instead of nuclear) remains valid without any change in the locally $p$-convex case (or even pseudoconvex, see 4.1 .

3.2. Diametral dimension. The notion of diametral dimension can be traced back to Pełczyński [15], using the $n$-dimensional diameters originally introduced by Kolmogorov [10].

$\left({ }^{1}\right)$ Not to be confused with the definition of [9, 19.7], which is different and applies only to normed spaces. 
Definition 4. Let $\mathcal{U}$ be a circled 0-basis of a topological linear space $E$. The diametral dimension of $E$ is the space

$$
\Delta(E):=\left\{\xi: \forall U \in \mathcal{U}, \exists V \in \mathcal{U}, V \subset U, \delta_{n}(V, U) \xi_{n} \rightarrow 0\right\}
$$

where

$\delta_{n}(V, U):=\inf \{\delta>0:$ there is a subspace $L$ of $E$, of dimension $\leq n$, such that $V \subset \delta U+L\}$.

Clearly $\Delta(E)$ does not depend on the choice of the 0-basis $\mathcal{U}$. Its size shows how "close" $E$ is to being finite-dimensional. For instance, Schwartz spaces can be characterized via this notion. Indeed, Jarchow's proof [9, 10.6.7] for locally convex spaces can be adapted to the locally $p$-convex case to show:

Proposition 3.2. For a locally p-convex space E, the following are equivalent:

(i) $E$ is Schwartz;

(ii) $l_{\infty} \subset \Delta(E)$;

(iii) $c_{0} \subsetneq \Delta(E)$.

In the same spirit, the connection with nuclearity was suggested by Gel'fand and proved by Mityagin [14].

TheOREM (Mityagin). For a locally convex space E, the following are equivalent:

(i) $E$ is nuclear;

(ii) there exists $\rho>0$ such that $n \mapsto n^{\rho} \in \Delta(E)$;

(iii) for all $\rho>0, n \mapsto n^{\rho} \in \Delta(E)$.

In view of this theorem, (ii) could be (and was in [11, 12, 18, 19]) taken as the definition of a nuclear space, making sense for locally $p$-convex spaces as well. But then Ligaud [12] proved something similar to Proposition 3.1. any locally $p$-convex space that is nuclear in this sense is necessarily locally convex.

So it seems that, although the definitions seem to be quite separated, nuclearity is indissociable from local convexity. The diametral dimensions, however, are pertinent in any convexity situation.

3.3. Diametral dimension of $S^{\nu}$. Back to our favorite multiscale sequence spaces. Taking for $U$ and $V$ the balls associated to the $p_{0}$-norms that were defined in (5), we can compute exactly the diameters $\delta_{n}(V, U)$ for some values of $n$. 
Lemma 3.3. If $0<\varepsilon^{\prime}<\varepsilon$ and $r, r^{\prime}>0, U:=B_{A, \varepsilon}(r)$ and $V:=$ $B_{A, \varepsilon^{\prime}}\left(r^{\prime}\right)$, then for $j_{0} \in \mathbb{N}_{0}$ and $n:=2^{j_{0}}-1$,

$$
\delta_{n}(V, U)=\frac{r^{\prime}}{r} 2^{\left(\varepsilon^{\prime}-\varepsilon\right) j_{0}} .
$$

The decrease of $\delta_{n}(V, U)$ with respect to $n$ immediately implies that for all $n \in \mathbb{N}_{0}$,

$$
\frac{r^{\prime}}{r}(n+1)^{\varepsilon^{\prime}-\varepsilon} \leq \delta_{n}(V, U) \leq \frac{r^{\prime}}{r}\left(\frac{n+1}{2}\right)^{\varepsilon^{\prime}-\varepsilon} .
$$

Proof of Lemma 3.3. Up to some scaling, we can suppose $r=r^{\prime}=1$. For short we shall write $\|x\|_{U}:=\|x\|_{A, \varepsilon},\|x\|_{V}:=\|x\|_{A, \varepsilon^{\prime}}$ and $\theta:=2^{\left(\varepsilon^{\prime}-\varepsilon\right) j_{0}}$.

First, let $L$ be the $n$-dimensional space of sequences $x$ such that $j \geq j_{0} \Rightarrow$ $x_{j, k}=0$. We claim that $V \subset \theta U+L$. Indeed, any $x \in V$ is the sum $x^{\prime}+x^{\prime \prime}$ where $x^{\prime \prime} \in L$ and the coefficients of $x^{\prime}$ at scales $j<j_{0}$ are all zero. By Lemma 2.1(i), $\left\|x^{\prime}\right\|_{U} \leq \theta$, thus $x^{\prime} \in \theta U$. We have proved that $\delta_{n}(V, U) \leq \theta$.

Now for the converse inequality: If it were not true, then there would exist $\theta^{\prime}>0$ such that $\delta_{n}(V, U)<\theta^{\prime}<\theta$ and a subspace $L$ of dimension $n$ such that $V \subset \theta^{\prime} U+L$. Let $P_{n+1}$ be the natural projection onto the first $n+1=2^{j_{0}}$ components, i.e. $\left\{(j, k): 0 \leq j<j_{0}, 0 \leq k<2^{j}\right\} \cup\left\{\left(j_{0}, 0\right)\right\}$. The range $P^{*}$ of this linear operator is finite-dimensional and strictly contains $L^{*}:=P_{n+1} L$. It follows that there is $x^{*} \in P^{*} \backslash L^{*}$ such that

$$
r_{0}:=\inf _{y \in L^{*}}\left\|x^{*}-y\right\|_{V}>0 .
$$

Since $1<\theta / \theta^{\prime}$, there is $y^{*} \in L^{*}$ such that

$$
r_{0} \theta / \theta^{\prime}>\left\|x^{*}-y^{*}\right\|_{V}=: s>0 .
$$

We have $\frac{1}{s}\left(x^{*}-y^{*}\right) \in P^{*} \cap V$, hence we can write

$$
\frac{1}{s}\left(x^{*}-y^{*}\right)=\theta^{\prime} X^{\prime}+X^{\prime \prime}=\theta^{\prime} x^{\prime}+x^{\prime \prime}
$$

with $x^{\prime \prime}:=P_{n+1} X^{\prime \prime} \in L^{*}$ and $x^{\prime}:=P_{n+1} X^{\prime} \in U \cap P^{*}$. Since $x^{\prime} \in P^{*}$, by Lemma 2.1(ii) we have

$$
\left\|x^{\prime}\right\|_{V} \leq \theta^{-1}\left\|x^{\prime}\right\|_{U}
$$

hence

$$
\left\|x^{*}-y^{*}-s x^{\prime \prime}\right\|_{V}=s \theta^{\prime}\left\|x^{\prime}\right\|_{V}<r_{0} \theta\left\|x^{\prime}\right\|_{V} \leq r_{0}\left\|x^{\prime}\right\|_{U} \leq r_{0},
$$

which contradicts the definition 10 of $r_{0}$ since $y^{*}+s x^{\prime \prime} \in L^{*}$.

Our main result is the following.

THEOREM 1. The diametral dimension $\Delta\left(S^{\nu}\right)$ is the space of all slowly increasing sequences $\xi$, i.e. such that for all $\lambda>0, n^{-\lambda} \xi_{n}$ is bounded. 
Proof. Let $\xi$ be a sequence increasing slower than any power of $n$ and let $U \in \mathcal{U}$. That is to say, $U=: B_{A, \varepsilon}(r)$ and we choose $V:=B_{A, \varepsilon / 3}(r)$. Then the right-hand side of 9 shows that $\xi_{n} \delta_{n}(V, U) \rightarrow 0$, hence $\xi \in \Delta\left(S^{\nu}\right)$.

Conversely, suppose that $\xi \in \Delta\left(S^{\nu}\right)$ and let $\lambda>0$ be fixed. We pick $(A, \varepsilon)$ as in Lemma 2.2 and let $U:=B_{A, \varepsilon}(1)$. Our hypothesis says that there exists $V=B_{A^{\prime}, \varepsilon^{\prime}}\left(r^{\prime}\right) \in \mathcal{U}$ such that $\xi_{n} \delta_{n}(V, U) \rightarrow 0$. The fact that $V \in \mathcal{U}$ implies that $\varphi_{A^{\prime}, \varepsilon^{\prime}}(\alpha) \geq \nu(\alpha)$ for all $\alpha$, hence

$$
\varphi_{A^{\prime}, \lambda+\varepsilon^{\prime}}(\alpha)=\varphi_{A^{\prime}, \varepsilon^{\prime}}(\alpha+\lambda) \geq \nu(\alpha+\lambda) \geq \varphi_{A, \varepsilon}(\alpha) .
$$

It follows that $U^{\prime}:=B_{A^{\prime}, \lambda+\varepsilon^{\prime}}(1)$ contains $U$, so using this time the left-hand side of $(9)$, we obtain

$$
r^{\prime}(n+1)^{-\lambda} \leq \delta_{n}\left(V, U^{\prime}\right) \leq \delta_{n}(V, U)
$$

and $\xi_{n} n^{-\lambda} \rightarrow 0$.

COROLlary 3.4. The spaces $S^{\nu}$ are Schwartz, but not nuclear.

REMARK. All spaces $S^{\nu}$ have the same diametral dimension, but they are not isomorphic if for instance their convexity indices are different.

\section{The case $p_{0}=0$}

4.1. Pseudoconvexity. We no longer assume $p_{0}>0$. Then the $S^{\nu}$ topology cannot anymore be described by a set of $p$-seminorms for a single value of $p$, as in 2.1. To see how a $p$-seminorms-based topology can still be used, let us first recall the original definition of the $S^{\nu}$ topology using not norms but distances. Define, for $\alpha \in \mathbb{R}$ and $\beta \in\{-\infty\} \cup[0,+\infty)$, $d_{\alpha, \beta}(x, y):=d_{\alpha, \beta}(x-y)$ where

$$
d_{\alpha, \beta}(x):=\inf \left\{C \geq 0: \forall j, \#\left\{\left|x_{j, k}\right| \geq C 2^{-\alpha j}\right\} \leq C 2^{\beta j}\right\}
$$

(agreeing that $2^{j \beta}:=0$ when $\beta=-\infty$ ) and the ancillary metric space

$$
E(\alpha, \beta):=\left\{x \in \mathbb{C}^{\Lambda}: d_{\alpha, \beta}(x)<\infty\right\} .
$$

Then it can be shown that for any sequence $\alpha_{n}$ dense in $\left(\alpha_{\min }-1 / 2, \infty\right)$ and any sequence $\varepsilon_{m} \searrow 0$ we have

$$
S^{\nu}=\bigcap_{n, m} E\left(\alpha_{n}, \nu\left(\alpha_{n}\right)+\varepsilon_{m}\right) .
$$

We also recall that by a result of [3] using the closed graph theorem, a complete metrizable topology on $S^{\nu}$ that is stronger than the product topology is necessarily unique, so we can safely talk of "the" $S^{\nu}$ topology.

The following definition is rather standard.

Definition 5. A topological linear space is said to be locally pseudoconvex if there exists a family of $r$-seminorms $(0<r \leq 1)$ defining the topology of the space. 
Indeed, as our next result shows, this is the case for $S^{\nu}$ if a minor extra condition is satisfied. We recall that we defined

$$
\alpha_{\min }:=\inf \{\alpha: \nu(\alpha) \geq 0\}
$$

Theorem 2. Assume that $\alpha_{\min }>-\infty$. Then $S^{\nu}$ is a metrizable pseudoconvex space.

Proof. We shall build a system of $p_{m, n}$-seminorms defining the $S^{\nu}$ topology and such that $p_{m, n} \rightarrow 0$ with $m \rightarrow \infty$ (uniformly in $n$ ). Once again let $\left(\alpha_{n}\right)$ and $\left(\varepsilon_{m}\right)$ be suitable sequences as in (11). The seminorms are defined on the sum spaces

$$
\|x\|_{\mathfrak{b}_{m, n}}:=\inf \left\{\left\|x^{\prime}\right\|_{b_{p_{m, n}, \infty}^{s_{m, n}}}+\left\|x^{\prime \prime}\right\|_{b_{\infty, \infty}^{\alpha_{n}}}: x=x^{\prime}+x^{\prime \prime}\right\}
$$

or

$$
\|x\|_{\mathfrak{b}_{m, n}}:=\|x\|_{b_{\infty, \infty}^{\alpha_{n}}}
$$

if $s_{m, n}=\infty$, with

$$
p_{m, n}:=\frac{\varepsilon_{m}}{2\left(\alpha_{n}-\alpha_{\min }+1\right)} \quad \text { and } \quad s_{m, n}:=\alpha_{n}+\frac{1-\nu\left(\alpha_{n}\right)-\varepsilon_{m}}{p_{m, n}}
$$

Let us assume for the moment that:

LEMMA 4.1. Each $p_{m, n}$-norm $\|\cdot\|_{\mathfrak{b}_{m, n}}$ is continuous on $S^{\nu}$.

On the other hand, it is immediate to see that $\|x\|_{\mathfrak{b}_{m, n}}<\infty$ implies $\nu_{x}(\alpha) \leq \nu\left(\alpha_{n}\right)+\varepsilon_{m}$ for any $\alpha<\alpha_{n}$, hence $\bigcap_{m, n} \mathfrak{b}_{m, n}=S^{\nu}$ and the projective limit topology $\tau_{1}$ on the intersection is stronger than the $S^{\nu}$ topology $\tau_{2}$. Thus the diagonal is closed in $\left(\bigcap_{m, n} \mathfrak{b}_{m, n}, \tau_{1}\right) \times\left(S^{\nu}, \tau_{2}\right)$. Both $\left(\bigcap_{m, n} \mathfrak{b}_{m, n}, \tau_{1}\right)$ and $\left(S^{\nu}, \tau_{2}\right)$ are complete metrizable spaces so we can use the closed graph theorem to show that the topologies are in fact equivalent.

Proof of Lemma 4.1. Fix $m, n \in \mathbb{N}$. Note that $S^{\nu}=S^{\nu} \cap b_{\infty, \infty}^{\alpha_{\min }-1}$ and that this space has a topology stronger than the one defined by the distance

$$
\delta_{m, n}(x):=\max \left(d_{\alpha_{n}, \nu\left(\alpha_{n}\right)+\varepsilon_{m} / 2}(x),\|x\|_{b_{\infty, \infty}^{\alpha_{\min }}-1}\right) .
$$

It suffices to show that $\delta_{m, n}(x) \rightarrow 0$ implies $\|x\|_{\mathfrak{b}_{m, n}} \rightarrow 0$.

Fix $x$ and let $C>\delta_{m, n}(x)$. On one hand, this means that there are, at any scale $j$, fewer than $C 2^{\left(\nu\left(\alpha_{n}\right)+\varepsilon_{m} / 2\right) j}$ coefficients with $\left|x_{j, k}\right| \geq C 2^{-\alpha_{n} j}$. We put those coefficients into $x^{\prime}$ and the rest into $x^{\prime \prime}$, so that $x=x^{\prime}+x^{\prime \prime}$. On the other hand, the coefficients of $x^{\prime}$ are all $\leq C 2^{-\left(\alpha_{\min }-1\right) j}$. 
If we compute the $b_{p_{m, n}, \infty}^{s_{m, n}}$ Besov norm of $x^{\prime}$ we obtain (writing for short $s:=s_{m, n}$ and $\left.p:=p_{m, n}\right)$

$$
\begin{aligned}
\left\|x^{\prime}\right\|_{b_{p, \infty}^{s}} & \leq \sup _{j \in \mathbb{N}_{0}} 2^{(s-1 / p) j}\left(C 2^{\left(\nu\left(\alpha_{n}\right)+\varepsilon_{m} / 2\right) j}\right)^{1 / p} C 2^{-\left(\alpha_{\min }-1\right) j} \\
& \leq \sup _{j \in \mathbb{N}_{0}} C^{(p+1) / p} 2^{\left(\alpha_{n}-\left(\nu\left(\alpha_{n}\right)+\varepsilon_{m}\right) / p\right) j} 2^{\frac{\nu\left(\alpha_{n}\right)+\varepsilon_{m} / 2}{p} j} 2^{-\left(\alpha_{\min }-1\right) j} \\
& \leq \sup _{j \in \mathbb{N}_{0}} C^{(p+1) / p} 2^{\left(\alpha_{n}-\alpha_{\min }+1-\varepsilon_{m} / 2 p\right) j} \leq C^{(p+1) / p} .
\end{aligned}
$$

As for $x^{\prime \prime}$, we see directly that

$$
\left\|x^{\prime \prime}\right\|_{b_{\infty, \infty}^{\alpha_{n}}} \leq C
$$

so finally $\|x\|_{\mathfrak{b}_{m, n}} \leq \delta_{m, n}(x)^{(p+1) / p}+\delta_{m, n}(x)$ and the continuity is proved.

Is the condition $\alpha_{\min }>-\infty$ necessary for pseudoconvexity? Well, almost. There is one very particular situation for which $\alpha_{\min }=-\infty$ and $S^{\nu}$ is still locally convex: when $\nu(\alpha)=1$ for all $\alpha \in \mathbb{R}$. In that case $S^{\nu}$ is simply the space $\mathbb{C}^{\Lambda}$ of all sequences, endowed with the product topology. But it is an exception. Indeed, we shall prove the following result.

Proposition 4.2. If $\alpha_{\min }=-\infty$ and $S^{\nu} \neq \mathbb{C}^{\Lambda}$ then $S^{\nu}$ is not locally pseudoconvex.

Before starting the proof, we first introduce some construction and notation. For each $\alpha \in \mathbb{R}$ and $\beta \in\{-\infty\} \cup[0,1]$, let $M^{\alpha, \beta}$ denote the set of sequences that have, at one particular scale $j$, not more than $2^{\beta j}$ non-zero coefficients, which are (in modulus) less than or equal to $2^{-\alpha j}$; and all coefficients at all other scales are zero. Now let $p>0$ and let $\|\cdot\|_{p}$ be an arbitrary continuous $p$-seminorm on $S^{\nu}$. We associate to it the function

$$
\mu: \alpha \mapsto \sup \left\{\beta \in\{-\infty\} \cup[0,1]: \sup \left\{\|x\|_{p}: x \in M^{\alpha, \beta}\right\}<\infty\right\} .
$$

Notice that for any $\alpha>\alpha_{\min }$ the set $M^{\alpha, 0}$ is bounded in $S^{\nu}$, hence

$$
\sup \left\{\|x\|_{p}: x \in M^{\alpha, 0}\right\}<\infty .
$$

We shall also prove later that $\mu$ has minimal slope $p$, more precisely:

LEMma 4.3. If $\|\cdot\|_{p}$ is a continuous p-seminorm on $S^{\nu}$, then for all $\alpha \in \mathbb{R}$ and all $\delta \geq 0$ we have $\mu(\alpha+\delta) \geq \min (\mu(\alpha)+p \delta, 1)$.

Proof of Proposition 4.2. If $S^{\nu} \neq \mathbb{C}^{\Lambda}$, then there is $\alpha_{1} \in \mathbb{R}$ such that $\nu\left(\alpha_{1}\right)<1$. Since $\nu$ is right-continuous, we can certainly choose $\varepsilon>0$ and $\delta>0$ such that $\nu\left(\alpha_{1}+\delta\right)+2 \varepsilon<1$. If we believe that $S^{\nu}$ is locally pseudoconvex, then there should exist some continuous $p$-seminorm $\|\cdot\|_{p}$ on $S^{\nu}$ such that for all $j$,

$$
\|x\|_{p} \leq 1 \Rightarrow \#\left\{x:\left|x_{j, k}\right| \geq 2^{-\left(\alpha_{1}+\delta\right) j}\right\} \leq 2^{\left(\nu\left(\alpha_{1}+\delta\right)+\varepsilon\right) j} .
$$


Let us consider the function $\mu$ defined in $(12)$, associated with this seminorm $\|\cdot\|_{p}$. We claim that $\mu\left(\alpha_{1}\right)<1$. Indeed, if not, then for every $\beta<1$ we have

$$
\sup \left\{\|x\|_{p}: x \in M^{\alpha_{1}, \beta}\right\}<\infty .
$$

In particular, since $\nu\left(\alpha_{1}+\delta\right)+2 \varepsilon<1$, there exists $R<\infty$ such that $\|x\|_{p} \leq R$ for each $x \in M^{\alpha_{1}, \nu\left(\alpha_{1}+\delta\right)+2 \varepsilon}$. Take a large scale $j$ such that $R 2^{-\delta j} \leq 1$ and define $x_{j, k}:=2^{-\alpha_{1} j}$ for $\left\lfloor 2^{\left(\nu\left(\alpha_{1}+\delta\right)+2 \varepsilon\right) j}\right\rfloor$ values of $k$, and zero elsewhere. By construction we have $x \in M^{\alpha_{1}, \nu\left(\alpha_{1}+\delta\right)+2 \varepsilon}$, thus $\left\|R^{-1} x\right\|_{p} \leq 1$, which contradicts (14).

Now, as a consequence of Lemma 4.3 , for any $\delta \geq 0$ we have $\mu\left(\alpha_{1}-\delta\right) \leq$ $\mu\left(\alpha_{1}\right)-p \delta$. In particular, there exists $\alpha_{0}>\alpha_{\min }$ such that $\mu\left(\alpha_{0}\right)<0$, in other words $\sup \left\{\|x\|_{p}: x \in M^{\alpha_{0}, 0}\right\}=\infty$, which contradicts 13 . So the $p$-seminorm $\|\cdot\|_{p}$ cannot be continuous on $S^{\nu}$.

Proof of Lemma 4.3. Notice that $\mu$ is non-decreasing and we can assume that $-\infty<\mu(\alpha)<1-p \delta$, otherwise the result is trivial.

First we assume that $\mu(\alpha)>0$ and take $0<\beta<\mu(\alpha)$. We want to prove that there exists $C<\infty$ depending only on $\alpha, \beta, \delta$ such that $\|x\|_{p} \leq C$ for each $x \in M^{\alpha+\delta, \beta+p \delta}$. Let us pick such an $x$ for which the non-zero scale is $j_{0}$. We can assume that $p \delta j_{0}>1$ and $\beta j_{0}>1$ since $\|x\|_{p}$ is bounded on the subset of $M^{\alpha+\delta, \beta+p \delta}$ for which $j_{0} \leq \max \left((p \delta)^{-1}, \beta^{-1}\right)$.

Let

$$
N:=\left\lfloor 2^{(\beta+p \delta) j_{0}}\right\rfloor, \quad M:=\left\lceil 2^{p \delta j_{0}}\right\rceil, \quad L:=\lfloor N / M\rfloor .
$$

We construct $M+1$ sequences $y^{i}$ based on $x$ as follows: first multiply $x$ by $2^{\delta j_{0}}$, then at scale $j_{0}$ split the (not more than $N$ ) non-zero coefficients $2^{\delta j_{0}} x_{j_{0}, k}$ into $M+1$ disjoint blocks, each of them having not more than $L \leq$ $2^{\beta j_{0}}$ coefficients with modulus $\left|y_{j_{0}, k}^{i}\right| \leq 2^{-\alpha j_{0}}$. The result of this construction is that each $y^{i}$ belongs to $M^{\alpha, \beta}$, hence $\sup _{i}\left\|y^{i}\right\|_{p} \leq C$ (because $\beta<\mu(\alpha)$ ) and finally

$$
\begin{aligned}
\|x\|_{p}^{p} & =\left\|\sum_{i} 2^{-\delta j_{0}} y_{i}\right\|_{p}^{p} \leq \sum_{i} 2^{-\delta p j_{0}}\left\|y^{i}\right\|_{p}^{p} \\
& \leq C^{p}(M+1) 2^{-\delta p j_{0}} \leq 2 C^{p} .
\end{aligned}
$$

In the case $\mu(\alpha)=0$ we proceed as previously but with $\beta=0$. The construction is very similar: take $N$ as before and $L=1$. The $N$ sequences $y^{i}$ now have at most one non-zero coefficient $\left|y_{j_{0}, k}^{i}\right| \leq 2^{-\alpha j_{0}}$. So each $y^{i}$ belongs to $M^{\alpha, 0}$, hence by $\sqrt{13}, \sup _{i}\left\|y^{i}\right\|_{p} \leq C$ and finally

$$
\begin{aligned}
\|x\|_{p}^{p} & =\left\|\sum_{i} 2^{-\delta j_{0}} y_{i}\right\|_{p}^{p} \leq \sum_{i} 2^{-\delta p j_{0}}\left\|y^{i}\right\|_{p}^{p} \\
& \leq C^{p} N 2^{-\delta p j_{0}} \leq C^{p} .
\end{aligned}
$$


4.2. An example by Ligaud. With a few modifications to the proof to accommodate the case $p_{0}=0$, it can be seen that Theorem 1 remains valid as long as $\alpha_{\min }>-\infty$. Proposition 3.2 also works; so $S^{\nu}$ is still a Schwartz space, even when it is only locally pseudoconvex. This gives us the occasion to revisit an example of Ligaud: in 12, he gives an explicit (but tricky and somewhat artificial) construction of a metrizable topological vector space which is a locally pseudoconvex non- $p$-convex Schwartz space. Actually, his example is a particular case of $S^{\nu}$.

Ligaud starts with a decreasing sequence $1 \geq p_{n} \rightarrow 0^{+}$and another sequence $\varepsilon_{n}>0$, which we shall assume for convenience to be summable. Then he ingeniously constructs a decreasing sequence of spaces $E_{n}$ such that (for $n \geq 2$ ) the following diagram commutes (horizontal "=" meaning isomorphism):

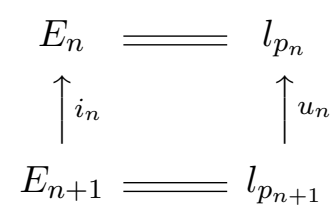

where $i_{n}$ is the inclusion, $l_{p_{n}}$ the standard $p_{n}$-normed space of sequences indexed by $\mathbb{N}$, and

$$
u_{n}:\left(\xi_{l}\right)_{l \in \mathbb{N}} \mapsto\left(\xi_{l} / l^{\varepsilon_{n}}\right)_{l \in \mathbb{N}} .
$$

We remark that each $u_{n}$, therefore each $i_{n}$, is compact, whence the Schwartz property holds for the projective limit $E:=\bigcap E_{n}$, which is indeed pseudoconvex but not $p$-convex.

If we now define $s_{n}:=-\sum_{l=n}^{\infty} \varepsilon_{l}$ and the Besov space $\mathfrak{l}_{n}:=b_{p_{n}, p_{n}}^{s_{n}+1 / p_{n}}$, then we have an isomorphism $\varphi_{n}$ from $l_{p_{n}}$ to $\mathfrak{l}_{n}$ which is explicitly given by

$$
\varphi_{n}:\left(\xi_{l}\right)_{l \in \mathbb{N}} \mapsto\left(x_{j, k}:=\frac{\xi_{2^{j}+k}}{\left(2^{j}+k\right)^{s_{n}}}\right)_{(j, k) \in \Lambda} .
$$

It is an isomorphism because

$$
\|\xi\|_{l_{p_{n}}} \leq\left\|\varphi_{n}(\xi)\right\|_{\mathfrak{l}_{n}}=\left(\sum_{j, k} 2^{j s_{n} p_{n}}\left|\frac{\xi_{2^{j}+k}}{\left(2^{j}+k\right)^{s_{n}}}\right|^{p_{n}}\right)^{1 / p_{n}} \leq 2^{-s_{n}}\|\xi\|_{l_{p_{n}}} .
$$

Furthermore, if $\mathfrak{i}_{n}$ represents the inclusion $\mathfrak{l}_{n+1} \hookrightarrow \mathfrak{l}_{n}$, the following diagram also commutes:

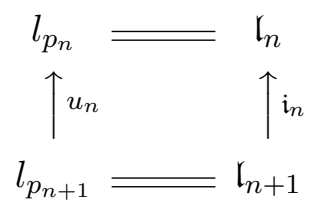

So we have for the projective limit

$$
E \simeq \bigcap_{n \geq 2} \mathfrak{l}_{n}=\bigcap_{n \geq 2} b_{p_{n}, \infty}^{s_{n}+1 / p_{n}}=S^{\nu}
$$


with $\nu(\alpha):=-\infty$ if $\alpha<0$ and $\nu(\alpha)=0$ if $\alpha \geq 0$. The first equality above holds because of standard Besov injections, the second one stems from the link between $S^{\nu}$ and Besov spaces (see [3]).

\section{References}

[1] J.-M. Aubry and F. Bastin, Advanced topology on the multiscale sequence spaces $S^{\nu}$, J. Math. Anal. Appl. 350 (2009), 439-454.

[2] J.-M. Aubry, F. Bastin and S. Dispa, Prevalence of multifractal functions in $S^{\nu}$ spaces, J. Fourier Anal. Appl. 13 (2007), 175-185.

[3] J.-M. Aubry, F. Bastin, S. Dispa and S. Jaffard, Topological properties of the sequence spaces $S^{\nu}$, J. Math. Anal. Appl. 321 (2006), 364-387.

[4] J.-M. Aubry and S. Jaffard, Random wavelet series, Comm. Math. Phys. 227 (2002), 483-514.

[5] K.-D. Bierstedt, R. G. Meise and W. H. Summers, Köthe sets and Köthe sequence spaces, in: Functional Analysis, Holomorphy and Approximation Theory (Rio de Janeiro, 1980), North-Holland Math. Stud. 71, North-Holland, Amsterdam, 1982, 27-91.

[6] C. Canus, J. Lévy Véhel and C. Tricot, Continuous large deviation multifractal spectrum: Definition and estimation, in: Fractals and Beyond (Valletta, 1998), World Sci., 1998, 117-128.

[7] S. Jaffard, Multifractal formalism for functions, I: results valid for all functions, SIAM J. Math. Anal. 28 (1997), 944-970.

[8] - Beyond Besov spaces, I: distributions of wavelet coefficients, J. Fourier Anal. Appl. 10 (2004), 221-246.

[9] H. Jarchow, Locally Convex Spaces, Teubner, Stuttgart, 1981.

[10] A. Kolmogoroff, Über die beste Annäherung von Funktionen einer gegebenen Funktionenklasse, Ann. of Math. (2) 37 (1936), 107-110.

[11] J. P. Ligaud, Sur les différentes définitions d'un espace nucléaire non localement convexe, Studia Math. 48 (1973), 257-269.

[12] —, Sur les rapports de convexité des topologies et bornologies dans les espaces nucléaires, ibid. 45 (1973), 181-190.

[13] C. Meneveau and K. Sreenivasan, Measurement of $f(\alpha)$ from scaling of histograms and applications to dynamical systems and fully developed turbulence, Phys. Lett. A 137 (1989), 103-112.

[14] B. S. Mityagin, Approximate dimension and bases in nuclear spaces, Russian Math. Surveys 16 (1961), 59-127; translated from Uspekhi Mat. Nauk 16 (1961), no. 4, 63-132.

[15] A. Pełczyński, On the approximation of S-spaces by finite dimensional spaces, Bull. Acad. Polon. Sci. Cl. III 5 (1957), 879-881.

[16] A. Pietsch, Nuclear Locally Convex Spaces, 2nd ed., Ergeb. Math. Grenzgeb. 66, Springer, 1972.

[17] R. H. Riedi, Multifractal processes, in: Theory and Applications of Long Range Dependence, Birkhäuser, 2003, 625-716.

[18] S. Rolewicz, Metric Linear Spaces, Monografie Mat. 56, PWN-Polish Sci. Publ., Warszawa, 1972. 
[19] S. Rolewicz, Open problems in theory of metric linear spaces, in: Actes du Colloque d'Analyse Fonctionnelle (Bordeaux, 1971), Bull. Soc. Math. France Mém. 31-32 (1972), 327-334.

Jean-Marie Aubry

Françoise Bastin

Lab. Anal. Math. Appl. (UMR CNRS 8050)

Université Paris-Est

61 av. du Général de Gaulle

F-94010 Créteil, France

E-mail: jmaubry@math.cnrs.fr 